\title{
Tandem Mass Spectrometry Detection of Quorum Sensing Activity in Multidrug Resistant Clinical Isolate Acinetobacter baumannii
}

\author{
Kok-Gan Chan, ${ }^{1}$ Huey Jia Cheng, ${ }^{1}$ Jian Woon Chen, ${ }^{1}$ Wai-Fong Yin, ${ }^{1}$ and Yun Fong Ngeow ${ }^{2}$ \\ ${ }^{1}$ Division of Genetics and Molecular Biology, Institute of Biological Sciences, Faculty of Science, University of Malaya, \\ 50603 Kuala Lumpur, Malaysia \\ ${ }^{2}$ Department of Microbiology, Faculty of Medicine, University of Malaya, 50603 Kuala Lumpur, Malaysia
}

Correspondence should be addressed to Kok-Gan Chan; kokgan@um.edu.my

Received 16 February 2014; Revised 1 June 2014; Accepted 2 June 2014; Published 2 July 2014

Academic Editor: Anna S. Levin

Copyright (c) 2014 Kok-Gan Chan et al. This is an open access article distributed under the Creative Commons Attribution License, which permits unrestricted use, distribution, and reproduction in any medium, provided the original work is properly cited.

Many Proteobacteria communicate via production followed by response of quorum sensing molecules, namely, $N$-acyl homoserine lactones (AHLs). These molecules consist of a lactone moiety with $N$-acyl side chain with various chain lengths and degrees of saturation at C-3 position. AHL-dependent QS is often associated with regulation of diverse bacterial phenotypes including the expression of virulence factors. With the use of biosensor and high resolution liquid chromatography tandem mass spectrometry, the AHL production of clinical isolate A. baumannii 4KT was studied. Production of short chain AHL, namely, $N$-hexanoylhomoserine lactone (C6-HSL) and N-octanoyl-homoserine lactone (C8-HSL), was detected.

\section{Introduction}

Acinetobacter spp. are Gram-negative bacteria widely found in natural environment, such as soil, water, and vegetation. Most species, however, have also been associated with a variety of human infections. In the hospital environment, they often colonize critically ill patients, particularly those in intensive care and burn units $[1,2]$. Furthermore, they cause a variety of opportunistic infections including bacteraemia, ventilator-associated pneumonia, surgical site infection, and catheter-related urinary tract infection [3]. Among them, A. baumannii has emerged as a highly troublesome pathogen for many institutions globally [4]. Since the 1970s, this bacterium has emerged as a formidable pathogen that is resistant to almost all currently available antibiotics via the acquisition of plasmids, transposons, and integrons bearing antibiotic resistance genes and the expression of numerous efflux pumps and porins [5]. The incidence of multidrug resistant infections has been increasing steadily, not just in hospital settings but also in the community [6]. Unfortunately, although the epidemiology of this pathogen has been extensively studied, relatively little is known about the molecular basis of its pathogenicity and virulence.

Quorum sensing (QS) is a mechanism of bacterial cellto-cell communication that relies on the production, sensing, and response to signaling molecules called autoinducers [7-9]. Arguably, the most widely studied QS molecule in Proteobacteria is $N$-acyl homoserine lactone (AHL) which is synthesized by an AHL synthase (LuxI homologue). AHL binds to its cognate receptor (LuxR homologue) [10, 11]. Once bound, this AHL-LuxR complex is activated to regulate QS-mediated gene expression [12]. Most Proteobacteria, including bacterial pathogens, rely on AHL to regulate QSdependent gene expression $[13,14]$. QS is of special interest because it regulates, inter alia, virulence determinants $[15,16]$ in a population-dependent manner [12]. Thus, characterizing AHL production represents the key step to understanding the molecular basis of virulence factors expression [17].

In this study, we have isolated clinical isolate $4 \mathrm{KT}$ as an aetiological agentfrom the sputum of an elderly patient with diabetes mellitus who was admitted for stroke management 
but developed a chest infection during her hospital stay in the year 2012. We have then identified the clinical isolate as $A$. baumannii using matrix-assisted laser desorption/ionizationtime-of-flight mass spectrometry (MALDI-TOF MS), and AHLs detection was our primary interest in order to study the QS activity in this clinical isolate. A variety of bacteria biosensors have been constructed $[18,19]$ to detect the presence of AHL signals by the activation of a reporter gene such as lacZ or lux or by the production or inhibition of a purple pigment in Chromobacterium violaceum or bioluminescence in lux-based Escherichia coli biosensors. However, the unequivocal identification of the type of AHLs produced requires analysis with high resolution analytical instruments such as mass spectrometry analysis. In the present work, high resolution liquid chromatography tandem mass spectrometry (LC-MS/MS) was used to detect the production of AHLs.

\section{Materials and Methods}

2.1. Bacteria, Biosensor, and Culture Conditions. C. violaceum CV026, Erwinia carotovora GS101, and E. carotovora PNP22 were grown aerobically in Luria Bertani (LB) broth with shaking $(220 \mathrm{rpm} / \mathrm{min})$ or on $\mathrm{LB}$ agar at $28^{\circ} \mathrm{C}[18,20]$. The components of LB medium were tryptone $(10 \mathrm{~g} / \mathrm{L})$, yeast extract $(5 \mathrm{~g} / \mathrm{L})$, and sodium chloride $(10 \mathrm{~g} / \mathrm{L})$ while Bacto agar $(15 \mathrm{~g} / \mathrm{L})$ was added to make LB agar. For MALDI-TOF MS analysis, clinical isolate $4 \mathrm{KT}$ was cultured on tryptic soy agar (TSA) (Scharlau, Spain) at $37^{\circ} \mathrm{C}$. The composition of the TSA includes casein peptone $(15 \mathrm{~g} / \mathrm{L})$, soy peptone $(5 \mathrm{~g} / \mathrm{L})$, sodium chloride $(5 \mathrm{~g} / \mathrm{L})$, and agar $(15 \mathrm{~g} / \mathrm{L})$.

2.2. Isolation and Identification of Clinical Isolate. Clinical isolate $4 \mathrm{KT}$ grew as non-lactose-fermenting colonies on MacConkey agar and was identified as Acinetobacter sp. by standard microbiological tests. This identification was subsequently confirmed as A. baumannii by MALDI-TOF MS analysis as described previously [21]. Briefly, by using formic acid-ethanol extraction method, clinical isolate $4 \mathrm{KT}$ was placed onto the MSP 96 target polished steel BC plate and subjected to Microflex MALDI-TOF (Bruker Daltonik $\mathrm{GmbH}$, Leipzig, Germany) bench-top mass spectrometer (equipped with UV laser at wavelength of $337 \mathrm{~nm}$ ) with the Bruker FlexControl software version 3.3 (Build 108). Routine antibiotic susceptibility testing with the agar disk diffusion (Kirby-Baur) test showed in vitro resistance to aminoglycosides, fluoroquinolones, first to fourth generations of cephalosporins, beta-lactam beta-lactamase inhibitor combinations, imipenem, cotrimoxazole, and tetracyclines. The only antibiotic showing some in vitro activity was polymyxin $B$.

2.3. Detection of N-Acyl Homoserine Lactones (AHLs) Synthesis in Clinical Isolate 4KT. The AHLs biosynthesis activity of clinical isolate $4 \mathrm{KT}$ was assayed by perpendicular streaking with biosensor CV026 on LB agar [18]. E. carotovora GS101 and E. carotovora PNP22 were used as positive control and negative control, respectively, in this study [20].

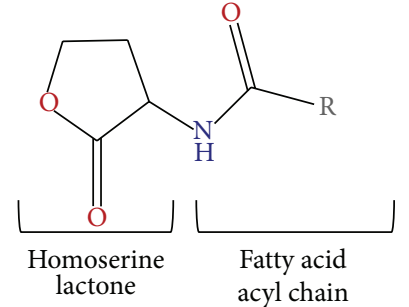

FIgURE 1: General structure of $N$-acyl homoserine lactone (AHL), where $\mathrm{R}$ represents the various acyl side chains.

2.4. AHL Extraction. Clinical isolate $4 \mathrm{KT}$ was grown overnight in $15 \mathrm{~mL}$ LB medium buffered with $50 \mathrm{mM} 3-[N$ morpholino] propanesulfonic acid (MOPS) at $\mathrm{pH} 6.5$ to prevent spontaneous lactonolysis of AHLs [22]. The supernatant was extracted twice with equal volumes of acidified ethyl acetate $(0.1 \% \mathrm{v} / \mathrm{v}$ glacial acetic acid $)$. Extract was concentrated to complete dryness under vacuum and resuspended with $1 \mathrm{~mL}$ of acetonitrile (ACN) for LC-MS/MS analysis.

2.5. Synthetic AHLs. All synthetic AHLs used in this procedure were purchased from commercial sources, Sigma Aldrich and Cayman (Michigan, USA). The structure of AHLs was shown in Figure 1. These AHLs included $\mathrm{N}$-butanoyl-homoserine lactone (C4-HSL), N-hexanoylhomoserine lactone (C6-HSL), $\mathrm{N}$-octanoyl-homoserine lactone (C8-HSL), N-decanoyl-homoserine lactone (C10HSL), $N$-dodecanoyl-homoserine lactone (C12-HSL), $N$ tetradecanoyl-homoserine lactone (C14-HSLs), $\mathrm{N}$-(3-oxohexanoyl)-homoserine lactone (3-oxo-C6-HSL), $N$-(3-oxohexanoyl)-homoserine lactone (3-oxo-C8-HSL), $\mathrm{N}$-(3-oxohexanoyl)-homoserine lactone (3-oxo-C10-HSL), N-(3oxododecanoyl)-homoserine lactone (3-oxo-C12-HSL), and $\mathrm{N}$-(3-oxotetradecanoyl)-homoserine lactone (3-oxo-C14HSL). AHL stock solutions used as standards were prepared by dissolving in ACN (Merck, Frankfurt, Germany) at a concentration of $1 \mathrm{~g} / \mathrm{L}$. The stock solutions were kept at $-20^{\circ} \mathrm{C}$ as standards in the LC-MS/MS analyses.

2.6. AHL Detection by LC-MS/MS. Separation of AHLs extract was performed with Agilent 1290 Infinity LC system (Agilent Technologies Inc., USA) with the HPLC column SB (C18 column, $2.1 \mathrm{~mm} \times 50 \mathrm{~mm}, 1.8 \mu \mathrm{m}$ particle size, Agilent Technologies Inc., USA). Sample elution was carried out for $15 \mathrm{~min}$ at a constant flow rate of $0.5 \mathrm{~mL} / \mathrm{min}$ at $37^{\circ} \mathrm{C}$ column temperature. The injection volume was at $2 \mu \mathrm{L}$. Mobile phase A was $0.1 \% \mathrm{v} / \mathrm{v}$ formic acid in ultrapure water and mobile phase $\mathrm{B}$ was $0.1 \% \mathrm{v} / \mathrm{v}$ formic acid in $\mathrm{ACN}$. The gradient profiles for the UPLC condition were fixed at (time: mobile phase A : mobile phase B) 0 min: $80: 20,7 \mathrm{~min}: 50: 50,12 \mathrm{~min}$ : $20: 80$, and 14 min: $80: 20$.

MS detection of the separated compounds from UPLC was conducted using the Agilent 6490 Triple Quadrupole LC/MS system (Agilent Technologies Inc., USA). The ion source involved electrospray ion (ESI) used in positive mode. Precursor ion experiment was performed (Figure 2) for the 


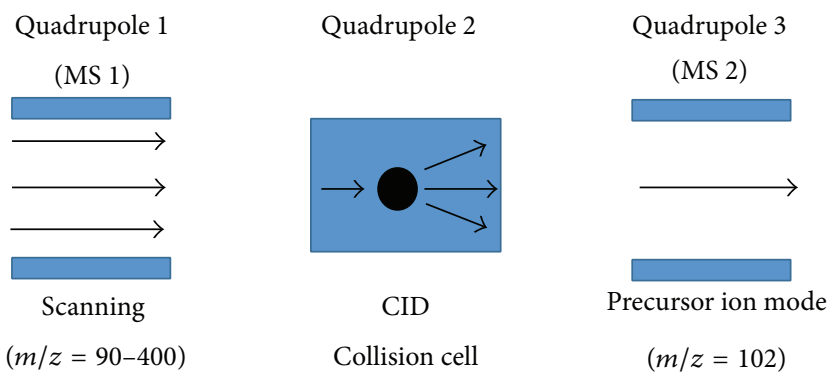

Figure 2: Precursor ion experiment using Agilent 6490 Triple Quadrupole LC/MS system.

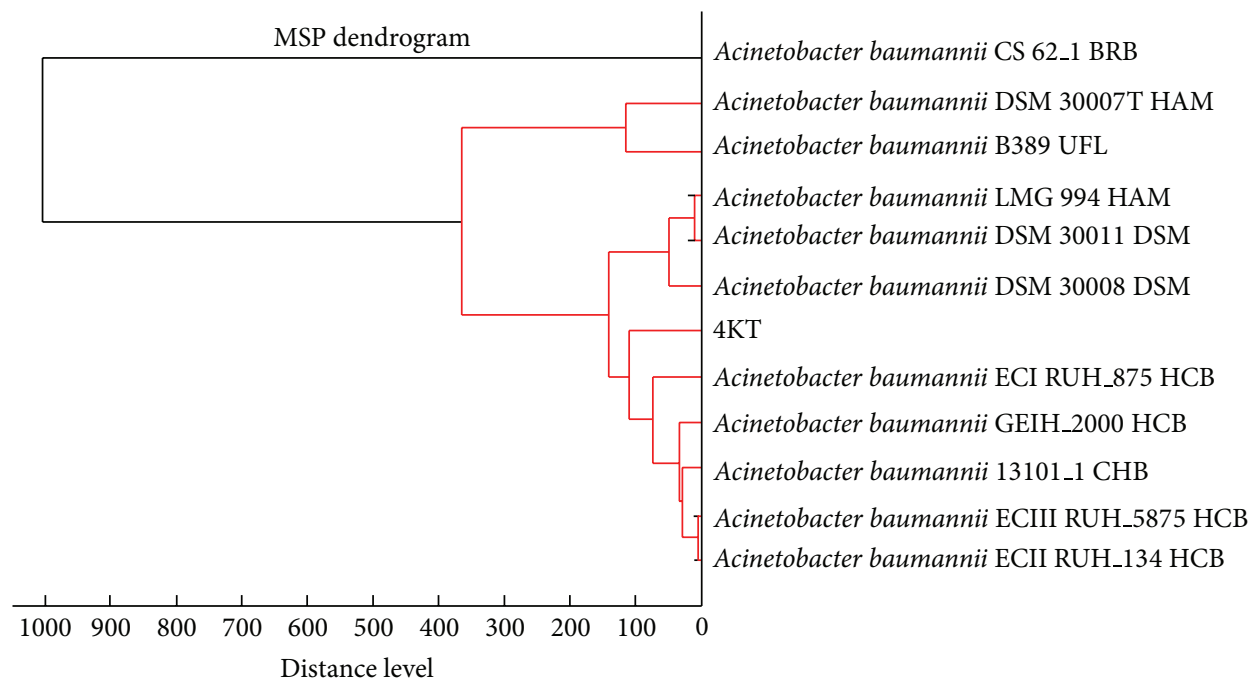

FIgURE 3: Score-oriented dendrogram of A. baumannii clinical isolate 4KT (labeled as $4 \mathrm{KT}$ in the dendrogram).

detection of AHLs in which the product ion $\mathrm{m} / z$ was set as 102 indicating the $[\mathrm{M}+\mathrm{H}]^{+}$ion of the core lactone ring moiety [23]. The $m / z$ value of the precursor ions was then scanned from 90 to 400 , thus allowing the identification of various AHLs based on the detection of the core HSL moiety fragmented in the collision cell. The Agilent MassHunter software was subsequently used for the MS data analysis. Analysis was performed by comparison of retention index and extracted ion (EI) mass spectra with synthetic AHL compounds.

\section{Results and Discussion}

A. baumannii is one of the most common opportunist pathogens in healthcare settings globally [4]. It appears to have a propensity for developing antimicrobial resistance and causing serious therapeutic problems. Diabetic patients and other immunocompromised individuals are at higher risk of developing infections by this organism than the rest of the population [24]. Clinical isolate $4 \mathrm{KT}$ from a diabetic patient with a hospital-acquired infection was identified as $A$. baumannii with a MALDI Biotyper score of 2.384 in the best match and 2.337 in the second best match. The score-oriented dendrogram of $4 \mathrm{KT}$ (Figure 3 ) clearly shows that this isolate belongs to the species $A$. baumannii.

QS activity in A. baumannii has been described previously [25]. Niu et al. (2008) [26] reported AHL synthase (AbaI) directed production of $\mathrm{N}$-(3-hydroxydodecanoyl)HSL (3-hydroxy-C12-HSL) along with other minor AHLs in an A. baumannii opportunistic pathogen. Besides that, Chan and coworkers [27] showed the production of 3-oxo-C12-HSL and 3-hydroxy-C12-HSL in an environmental Acinetobacter sp. strain GG2. In this study, preliminary QS screening with biosensor CV026 indicated 4KT did not produce short chain AHLs since no purple pigmentation was observed (data not shown). However, using LC-MS/MS, C6-HSL ( $m / z$ 200.400), and C8-HSL $(m / z 228.500)$ was detected from AHLs extract from $4 \mathrm{KT}$ (Figure 4). Though many AHLs profiles of $A$. baumannii have been studied previously; to the best of our knowledge, this is the first report of AHL profiles in a multidrug resistant $A$. baumannii.

Discrepancy in AHL production profile in different bacterial strains belonging to the same species is not peculiar to A. baumannii. It has been reported that nosocomial Acinetobacter clinical isolates showed a distinct induction pattern that is different from the AHL profile of environmental isolates of A. calcoaceticus [28]. This difference of 


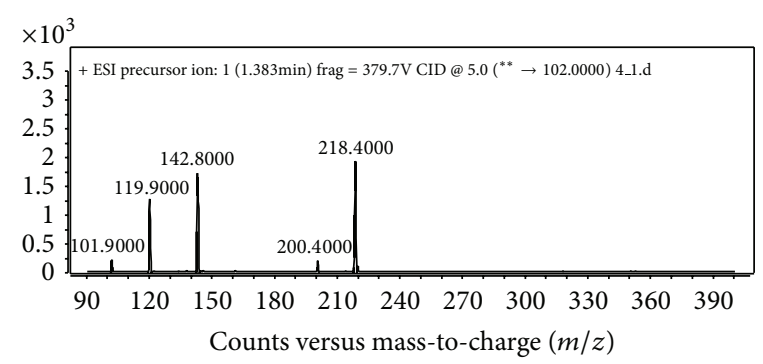

(a)

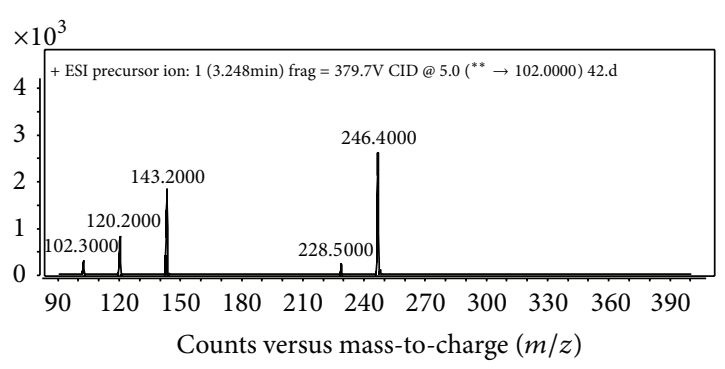

(b)

FIGURE 4: Mass spectrometry analysis of AHL produced by A. baumannii clinical isolate 4KT. (a) C6-HSL: retention time: 1.144 min; $m / z$ 200.400; abundance: 377.04; abundance\%: 17.98. (b) C8-HSL: retention time: $3.105 \mathrm{~min} ; \mathrm{m} / \mathrm{z} 228.100$; abundance: 236.74 ; abundance\%: 13.44 .

AHL production profile could also be due to the composition of the bacterial growth medium used and the duration of incubation [25]. It has been reported that bacteria cultured in minimal medium displayed one or two more signals, as compared to complex medium, and most bacteria showed AHL production in the stationary phase, perhaps due to the highest population cell density at this stage [25]. Besides, Middleton and coworkers [29] had proven that the AHL production profile showed variation for AHLs isolated directly from the sputum samples from patients comparing with AHLs extracted from in vitro analysis. In our study, the AHLs were extracted in vitro from isolated A. baumannii.

Recently, many researchers have reported on the potential use of QS as a target for the development of anti-infective therapy [30-37]. The discovery of AHLs production in multidrug resistant bacteria is important as it opens another therapy option in addition to normal antibiotic treatment which is ineffective in multidrug resistant bacteria. Quorum quenching, or interference of QS, has gained much attention due to its nonantibiotic application to attenuate bacterial infections [38-40]. The identification of AHLs in pathogens such as A. baumannii clinical isolate $4 \mathrm{KT}$ will allow screening for biomimetic or chemical analogs that can block QS mechanisms. As these molecules are mostly not antibiotics, they will not induce antibiotic resistance. This approach has been taken by Stacy and coworkers [34] who used nonnative ligands containing an aromatic aryl group closely similar to the 3-hydroxy-C12-HSL that blocks the AHL QS system in A. baumannii. The AHLs (C6-HSL and C8-HSL) produced by $A$. baumannii clinical isolate $4 \mathrm{KT}$ represent another group of AHLs that may be used for the discovery of nonnative ligands that can act as novel therapeutic drugs. This will hopefully lead to a wider spectrum of anti-QS drugs for the treatment of infections by multidrug resistant $A$. baumannii. Quorum quenching, however, only interrupts the QS activity among the bacteria and does not kill the bacteria, hence minimizing the multidrug resistance pressure; thus the approach should be applied together with the use of other drugs for synergistic effects to combat multidrug resistant pathogens.

\section{Conclusions}

Our work has shown the detection of QS activity in a multidrug resistant $A$. baumannii clinical isolate $4 \mathrm{KT}$ by using high resolution mass spectrometry. The production of QS signaling molecules, C6-HSL and C8-HSL, in this clinical isolate was found. This work will facilitate the search for QSmediated genes in this pathogen and possibly pave the way for the development of anti-QS drugs to combat multidrug resistant A. baumannii.

\section{Conflict of Interests}

The authors declare that there is no conflict of interests regarding the publication of this paper.

\section{Acknowledgment}

This work was supported by a High Impact Research Grant (Bacteria Quorum Quenching HIR-MOHE Grant: UM.C/625/1/HIR/MOHE/CHAN/01, A000001-50001) provided by the University of Malaya to Kok-Gan Chan, which is gratefully acknowledged. Yun Fong Ngeow thanks the financial support from High Impact Research Grant (Nature Microbiome HIR-MOHE Grant: UM.C/ 625/1/HIR/MOHE/CHAN/14/4, H50001-00-A000038).

\section{References}

[1] A. E. Simor, M. Lee, M. Vearncombe et al., "An outbreak due to multiresistant Acinetobacter baumannii in a burn unit: risk factors for acquisition and management," Infection Control and Hospital Epidemiology, vol. 23, no. 5, pp. 261-267, 2002.

[2] E. G. Playford, J. C. Craig, and J. R. Iredell, "Carbapenemresistant Acinetobacter baumannii in intensive care unit patients: risk factors for acquisition, infection and their consequences," Journal of Hospital Infection, vol. 65, no. 3, pp. 204-211, 2007.

[3] M.-L. Joly-Guillou, "Clinical impact and pathogenicity of Acinetobacter," Clinical Microbiology and Infection, vol. 11, no. 11, pp. 868-873, 2005. 
[4] A. Y. Peleg, H. Seifert, and D. L. Paterson, "Acinetobacter baumannii: emergence of a successful pathogen," Clinical Microbiology Reviews, vol. 21, no. 3, pp. 538-582, 2008.

[5] J. Vila, S. Martí, and J. Sánchez-Céspedes, "Porins, efflux pumps and multidrug resistance in Acinetobacter baumannii," Journal of Antimicrobial Chemotherapy, vol. 59, no. 6, pp. 1210-1215, 2007.

[6] L. Dijkshoorn, A. Nemec, and H. Seifert, "An increasing threat in hospitals: multidrug-resistant Acinetobacter baumannii," Nature Reviews Microbiology, vol. 5, no. 12, pp. 939-951, 2007.

[7] C. Fuqua, S. C. Winans, and E. P. Greenberg, "Census and consensus in bacterial ecosystems: the LuxR-LuxI family of quorum-sensing transcriptional regulators," Annual Review of Microbiology, vol. 50, pp. 727-751, 1996.

[8] C. Fuqua and E. P. Greenberg, "Self perception in bacteria: quorum sensing with acylated homoserine lactones," Current Opinion in Microbiology, vol. 1, no. 2, pp. 183-189, 1998.

[9] P. Williams, K. Winzer, W. C. Chan, and M. Cámara, "Look who's talking: communication and quorum sensing in the bacterial world," Philosophical Transactions of the Royal Society B: Biological Sciences, vol. 362, no. 1483, pp. 1119-1134, 2007.

[10] C. Fuqua, M. R. Parsek, and E. P. Greenberg, "Regulation of gene expression by cell-to-cell communication: acyl-homoserine lactone quorum sensing," Annual Review of Genetics, vol. 35, pp. 439-468, 2001.

[11] S. Swift, J. Allan Downie, N. A. Whitehead, A. M. Barnard, G. P. Salmond, and P. Williams, "Quorum sensing as a population-density-dependent determinant of bacterial physiology," Advances in Microbial Physiology, vol. 45, pp. 199-270, 2001.

[12] S. Swift, J. P. Throup, P. Williams, G. P. C. Salmond, and G. S. A. B. Stewart, "Quorum sensing: a population-density component in the determination of bacterial phenotype," Trends in Biochemical Sciences, vol. 21, no. 6, pp. 214-219, 1996.

[13] H. Withers, S. Swift, and P. Williams, "Quorum sensing as an integral component of gene regulatory networks in gramnegative bacteria," Current Opinion in Microbiology, vol. 4, no. 2, pp. 186-193, 2001.

[14] P. Williams, "Quorum sensing, communication and crosskingdom signalling in the bacterial world," Microbiology, vol. 153, no. 12, pp. 3923-3938, 2007.

[15] P. Williams, M. Camara, A. Hardman et al., "Quorum sensing and the population-dependent control of virulence," Philosophical Transactions of the Royal Society B: Biological Sciences, vol. 355, no. 1397, pp. 667-680, 2000.

[16] K. Winzer and P. Williams, "Quorum sensing and the regulation of virulence gene expression in pathogenic bacteria," International Journal of Medical Microbiology, vol. 291, no. 2, pp. 131$143,2001$.

[17] K. W. Hong, C. L. Koh, C. K. Sam, W. F. Yin, and K. G. Chan, "Quorum quenching revisited-from signal decays to signalling confusion," Sensors, vol. 12, no. 4, pp. 4661-4696, 2012.

[18] K. H. McClean, M. K. Winson, L. Fish et al., "Quorum sensing and Chromobacterium violaceum: exploitation of violacein production and inhibition for the detection of $\mathrm{N}$-acylhomoserine lactones," Microbiology, vol. 143, no. 12, pp. 3703-3711, 1997.

[19] M. K. Winson, S. Swift, L. Fish et al., "Construction and analysis of luxCDABE-based plasmid sensors for investigating $\mathrm{N}$-acyl homoserine lactone-mediated quorum sensing," FEMS Microbiology Letters, vol. 163, no. 2, pp. 185-192, 1998.
[20] S. McGowan, M. Sebaihia, S. Jones et al., "Carbapenem antibiotic production in Erwinia carotovora is regulated by CarR, a homologue of the LuxR transcriptional activator," Microbiology, vol. 141, no. 3, pp. 541-550, 1995.

[21] A. Mellmann, J. Cloud, T. Maier et al., "Evaluation of matrixassisted laser desorption ionization-time-of-flight mass spectrometry in comparison to $16 \mathrm{~S}$ rRNA gene sequencing for species identification of nonfermenting bacteria," Journal of Clinical Microbiology, vol. 46, no. 6, pp. 1946-1954, 2008.

[22] E. A. Yates, B. Philipp, C. Buckley et al., "N-acylhomoserine lactones undergo lactonolysis in a $\mathrm{pH}$-, temperature-, and acyl chain length-dependent manner during growth of Yersinia pseudotuberculosis and Pseudomonas aeruginosa," Infection and Immunity, vol. 70, no. 10, pp. 5635-5646, 2002.

[23] T. A. Gould, J. Herman, J. Krank, R. C. Murphy, and M. E. A. Churchill, "Specificity of acyl-homoserine lactone synthases examined by mass spectrometry," Journal of Bacteriology, vol. 188, no. 2, pp. 773-783, 2006.

[24] A. A. Alsultan, A. Hamouda, B. A. Evans, and S. G. B. Amyes, "Acinetobacter baumannii: emergence of four strains with novel bla OXA-51-like genes in patients with diabetes mellitus," Journal of Chemotherapy, vol. 21, no. 3, pp. 290-295, 2009.

[25] R. H. González, A. Nusblat, and B. Nudel, "Detection and characterization of quorum sensing signal molecules in Acinetobacter strains," Microbiological Research, vol. 155, no. 4, pp. 271-277, 2001.

[26] C. Niu, K. M. Clemmer, R. A. Bonomo, and P. N. Rather, "Isolation and characterization of an autoinducer synthase from Acinetobacter baumannii," Journal of Bacteriology, vol. 190, no. 9, pp. 3386-3392, 2008.

[27] K. Chan, S. Atkinson, K. Mathee et al., "Characterization of $\mathrm{N}$-acylhomoserine lactone-degrading bacteria associated with the Zingiber officinale (ginger) rhizosphere: co-existence of quorum quenching and quorum sensing in Acinetobacter and Burkholderia," BMC Microbiology, vol. 11, article 51, 2011.

[28] D. M. Sengstock, R. Thyagarajan, J. Apalara, A. Mira, T. Chopra, and K. S. Kaye, "Multidrug-resistant Acinetobacter baumannii: an emerging pathogen among older adults in community hospitals and nursing homes," Clinical Infectious Diseases, vol. 50, no. 12, pp. 1611-1616, 2010.

[29] B. Middleton, H. C. Rodgers, M. Cámara, A. J. Knox, P. Williams, and A. Hardman, "Direct detection of Nacylhomoserine lactones in cystic fibrosis sputum," FEMS Microbiology Letters, vol. 207, no. 1, pp. 1-7, 2002.

[30] M. Manefield, T. B. Rasmussen, M. Henzter et al., "Halogenated furanones inhibit quorum sensing through accelerated LuxR turnover," Microbiology, vol. 148, no. 4, pp. 1119-1127, 2002.

[31] T. B. Rasmussen and M. Givskov, "Quorum-sensing inhibitors as anti-pathogenic drugs," International Journal of Medical Microbiology, vol. 296, no. 2-3, pp. 149-161, 2006.

[32] Y. M. Chong, W. F. Yin, C. Y. Ho et al., "Malabaricone C from Myristica cinnamomea exhibits anti-quorum sensing activity," Journal of Natural Products, vol. 74, no. 10, pp. 2261-2264, 2011.

[33] T. Krishnan, W. F. Yin, and K. G. Chan, "Inhibition of quorum sensing-controlled virulence factor production in pseudomonas aeruginosa PAO1 by ayurveda spice clove (syzygium aromaticum) bud extract," Sensors, vol. 12, no. 4, pp. 4016-4030, 2012.

[34] D. M. Stacy, M. A. Welsh, P. N. Rather, and H. E. Blackwell, "Attenuation of quorum sensing in the pathogen Acinetobacter baumannii using non-native $N$-acyl homoserine lactones," ACS Chemical Biology, vol. 7, no. 10, pp. 1719-1728, 2012. 
[35] L. Y. Tan, W. Yin, and K. Chan, "Silencing quorum sensing through extracts of melicope lunu-ankenda," Sensors, vol. 12, no. 4, pp. 4339-4351, 2012.

[36] C. L. Koh, C. K. Sam, W. F. Yin et al., "Plant-derived natural products as sources of anti-quorum sensing compounds," Sensors, vol. 13, no. 5, pp. 6217-6228, 2013.

[37] S. N. Maisarah Norizan, W. F. Yin, and K. G. Chan, "Caffeine as a potential quorum sensing inhibitor," Sensors, vol. 13, no. 4, pp. 5117-5129, 2013.

[38] K. G. Chan, W. F. Yin, C. K. Sam, and C. L. Koh, "A novel medium for the isolation of $\mathrm{N}$-acylhomoserine lactonedegrading bacteria," Journal of Industrial Microbiology and Biotechnology, vol. 36, no. 2, pp. 247-251, 2009.

[39] K. G. Chan, C. S. Wong, W. F. Yin, C. K. Sam, and C. L. Koh, "Rapid degradation of $N$-3-oxo-acylhomoserine lactones by a Bacillus cereus isolate from Malaysian rainforest soil," Antonie van Leeuwenhoek, vol. 98, no. 3, pp. 299-305, 2010.

[40] C. S. Wong, W. F. Yin, Y. M. Choo, C. K. Sam, C. L. Koh, and K. G. Chan, "Coexistence of quorum-quenching and quorumsensing in tropical marine Pseudomonas aeruginosa strain MW3A," World Journal of Microbiology and Biotechnology, vol. 28, no. 2, pp. 453-461, 2012. 

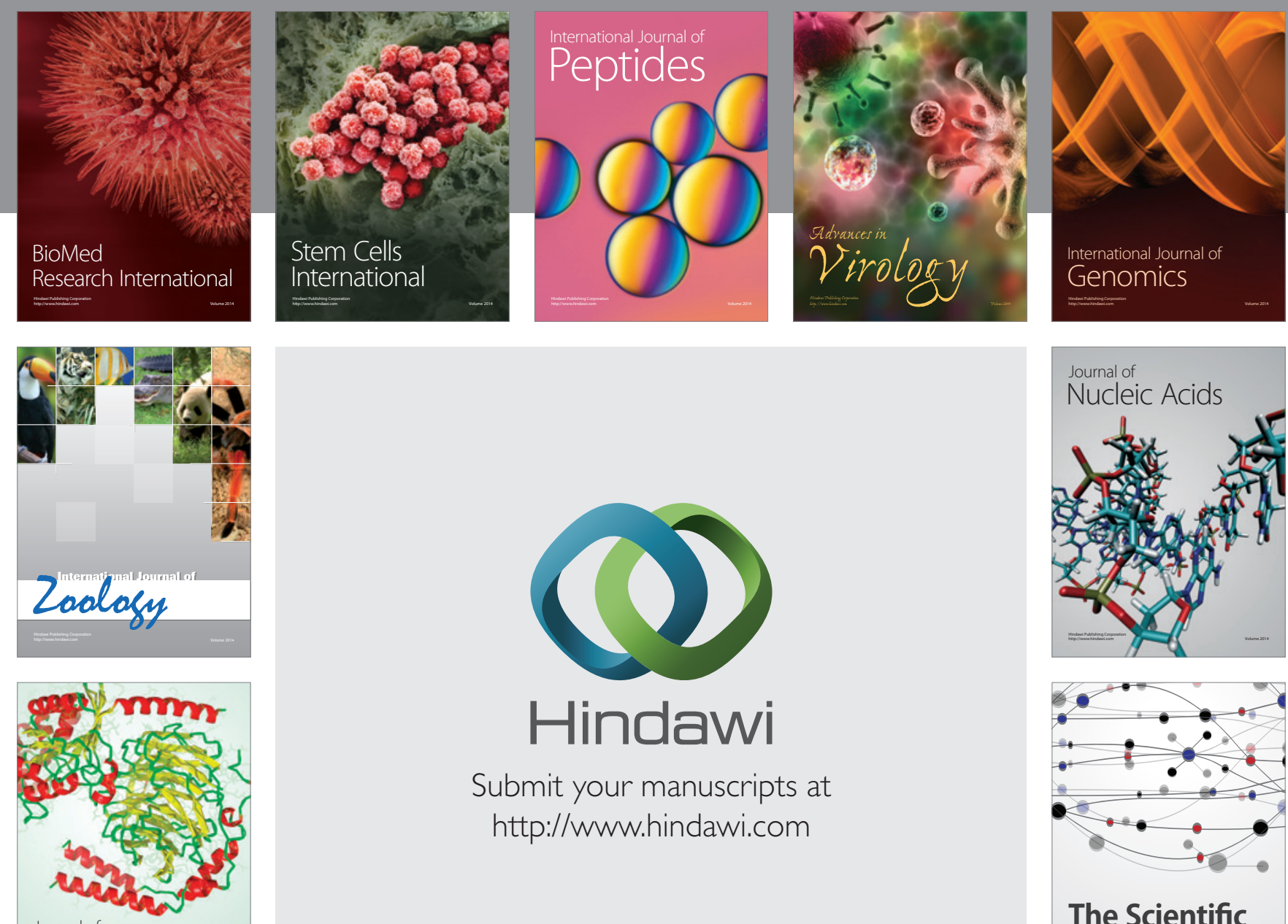

Submit your manuscripts at

http://www.hindawi.com

Journal of
Signal Transduction
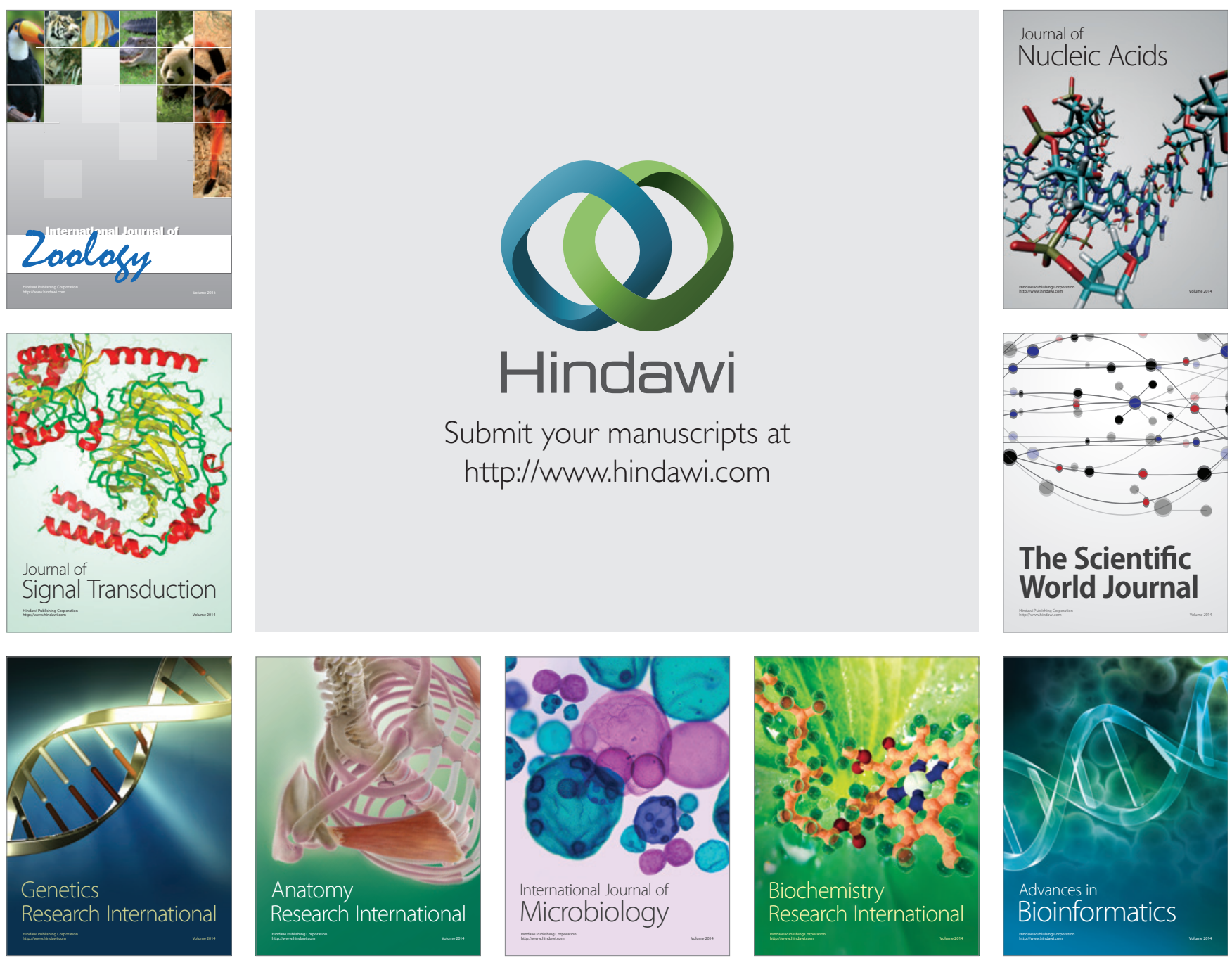

The Scientific World Journal
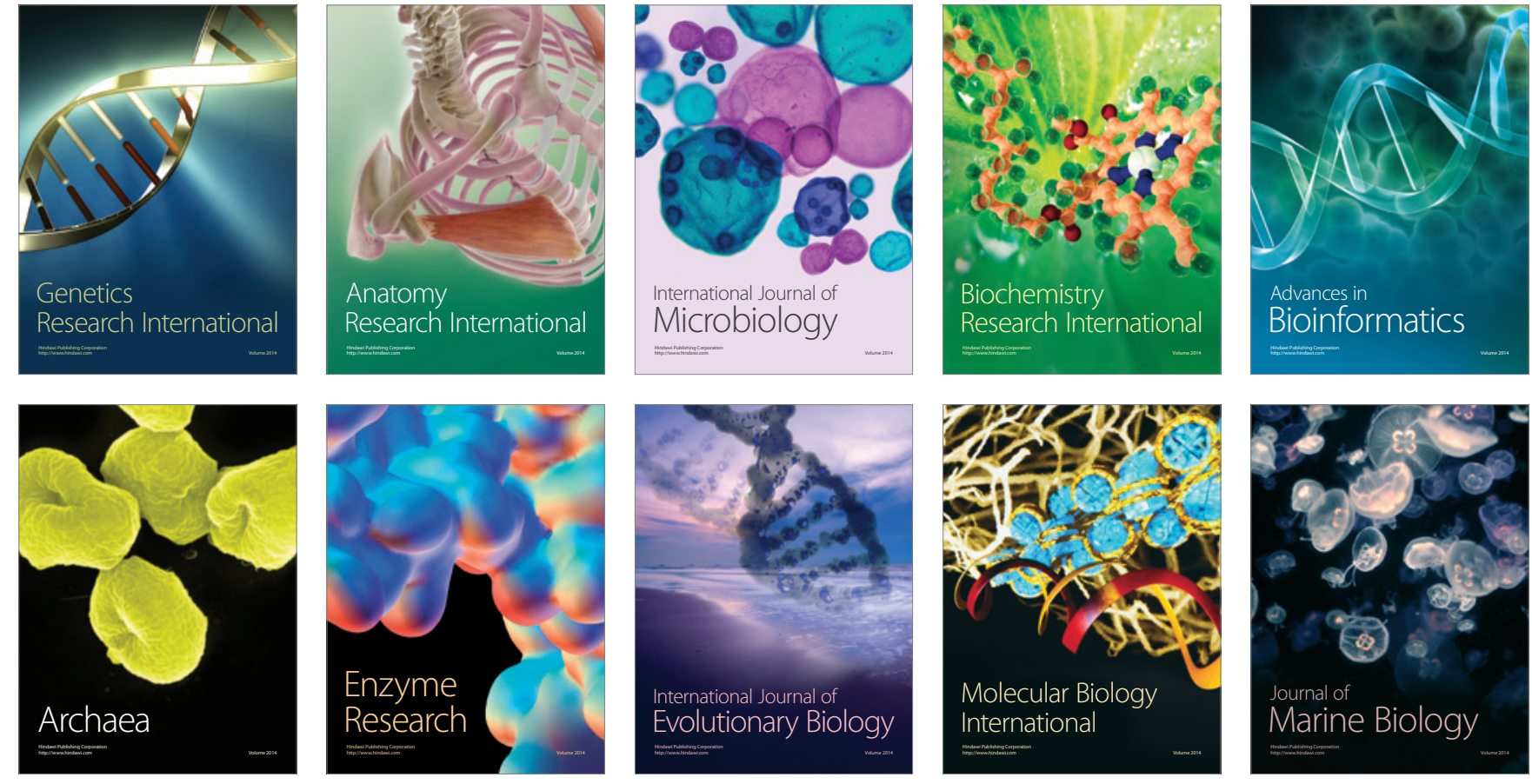